\title{
Influência da gordura corporal no desempenho do salto com con- tramovimento em judocas de diferentes categorias de peso
}

http://dx.doi.org/10.11606/1807-5509201700020345

\author{
Marina Saldanha da Silva ATHAYDE* \\ Daniele DETANICO* \\ Rafael Lima KONS*
}

Universidade Federal de Santa Catarina, Florianópolis, SC, Brasil.

\section{Resumo}

Este estudo teve como objetivo verificar a influência da gordura corporal nos parâmetros cinéticos do salto vertical em judocas de diferentes categorias. Participaram deste estudo 48 judocas do sexo masculino agrupados por categorias: 55-60 kg/60-66 kg (ligeiro/meio-leve), 66-73 kg/73-81 kg (leve/meio-médio) e 81-90 kg/90-100 kg (médio/meio-pesado). Os judocas realizaram saltos verticais countermovement jump (CMJ) sobre uma plataforma de força, sendo analisadas as variáveis de desempenho (altura do salto e potência), força máxima e pico de velocidade. Para análise dos dados foi utilizada análise de variância (ANOVA) e análise de covariância (ANCOVA) com o percentual de gordura como covariável. Para todos os testes foi utilizado $p<0,05$. Os principais resultados mostraram que os atletas de categorias mais pesadas (81-90 kg/90-100 kg) apresentaram maior percentual de gordura $(p<0,05)$ e somatório de dobras $(p<$ $0,05)$ quando comparado às demais categorias $(55-60 \mathrm{~kg} / 60-66 \mathrm{~kg}$ e 66-73 kg/73-81 kg). Foi encontrado menor desempenho nos judocas mais pesados em quase todas as variáveis do CMJ quando comparados com judocas de categorias mais leves, com destaque para altura do salto $(55-60 \mathrm{~kg} / 60-66 \mathrm{~kg}=46,78 \pm$ $6,05 \mathrm{~cm} ; 66-73 \mathrm{~kg} / 73-81 \mathrm{~kg}=47,42 \pm 4,39 \mathrm{~cm} ; 81-90 \mathrm{~kg} / 90-100 \mathrm{~kg}=42,11 \pm 4,11 ; p<0,05)$. Porém, quando retirada a influência do percentual de gordura as diferenças desapareceram para a maioria das variáveis $(p>0,05)$. Pode-se concluir que judocas de categorias mais leves apresentaram valores superiores na maioria das variáveis do CMJ (altura do salto, potência e velocidade) quando comparados com atletas de categorias mais pesadas. Esses resultados parecem ser influenciados pelo menor percentual de gordura dos judocas mais leves.

PalAVRas-Chave: Esportes de combate; Ciclo alongamento-encurtamento; Salto com contra movimento; Potência muscular; Composição corporal.

\section{Introdução}

O judô é considerado um esporte aberto e acíclico, no qual variáveis táticas e técnicas ${ }^{1}$, morfológi$\mathrm{cas}^{2}$, fisiológicas ${ }^{3}$ e psicológicas ${ }^{4}$ podem determinar o resultado da luta. Do ponto de vista fisiológico, o judô é caracterizado como uma modalidade intermitente de alta intensidade (20-30 s intercalados com 5-10 s de pausa) $)^{5}$. Os atletas precisam realizar um elevado número de ações explosivas, como entrada de golpe, disputa de pegada e transição para o solo durante o combate, o qual tem 4 minutos cronometrados (masculino). Essas características estruturais exigem elevada demanda física do atleta para suportar o tempo e a intensidade da luta5.
Tem sido observado que durante as lutas judô, os atletas passam cerca de $50 \%$ do tempo em disputas de pegada, com o objetivo de dominar o adversário, perturbar o equilíbrio e executar uma técnica de projeçáo ${ }^{7}$. No entanto, tendo em vista as regras atuais, pode-se sugerir maior volume e entrada de golpes, muitas vezes sem a intenção de projetar, apenas para provocar a penalização em seu adversário e consequentemente ganhar a luta. $\mathrm{Na}$ execução dos golpes de projeção no judô, observa-se açóes explosivas dos membros inferiores ${ }^{5}$ e, muitas vezes, envolvendo o mecanismo do ciclo alongamento-encurtamento (CAE), por 
exemplo, em técnicas que necessitam do apoio do quadril para realizar o golpe ${ }^{8}$ (por exemplo, seoi-nage, harai-goshi, o-goshi). Um dos métodos mais confiáveis de estimar a potência muscular em ações que envolvem o CAE é utilizar o salto vertical com contramovimento $(\mathrm{CMJ})^{9}$. Especificamente no judô, o desempenho no CMJ tem sido associado com maior número de arremessos em teste específico do judô (Special Judo Fitness Test - SJFT) ${ }^{8}$.

O salto vertical, assim como outras variáveis neuromusculares, é influenciado pelas características antropométricas dos atletas ${ }^{10}$. Especificamente no judô, Detanico et al. ${ }^{11}$ verificaram que quanto maior a massa corporal dos atletas, maiores foram os valores absolutos de força máxima e potência pico obtidos no CMJ. Por outro lado, quanto menor a massa corporal maior foi a altura do salto e potência relativa no CMJ, indicando que, em termos gerais, judocas mais leves tendem a ser mais potentes e menos fortes, ao passo que judocas mais pesados tendem a ser mais fortes e menos potentes. Vale ressaltar que no estudo de Detanico et al. ${ }^{11}$ a amostra foi composta por judocas de não-elite, $o$ que pode ter influenciado os resultados. Além disso, neste estudo náo foi considerado o percentual de gordura corporal dos atletas, uma vez que tem sido reportada correlação positiva entre percentual de gordura e categoria de peso do judô ${ }^{2}$.

O elevado percentual de gordura dos atletas tem mostrado influência negativa no desempenho em açóes específicas do judô, uma vez que foi observada relação inversa entre percentual de gordura e número de arremessos em teste específico $(\mathrm{SJFT})^{12}$. Além disso, em outro estudo ${ }^{13}$

\section{Método}

\section{Participantes}

Participaram do estudo 48 judocas do sexo masculino com idade 21,6 \pm 4,6 anos divididos por categorias de peso agrupadas da seguinte maneira: $55-60$ kg/60-66 kg (ligeiro/meio-leve) ( $\mathrm{n}=19), 66-73 \mathrm{~kg} / 73-81 \mathrm{~kg}$ (leve/meio-médio) ( $\mathrm{n}=17)$ e $81-90 \mathrm{~kg} / 90-100 \mathrm{~kg}$ (médio/meio-pesado) $(\mathrm{n}=12)$. O tempo de prática no judô dos atletas da categoria $55-60 \mathrm{~kg} / 60-66 \mathrm{~kg}$ foi $8,6 \pm$ 5,8 anos; da categoria $66-73 \mathrm{~kg} / 73-81 \mathrm{~kg}$ foi 7,4 $\pm 4,4$ anos e da categoria $81-90 \mathrm{~kg} / 90-100 \mathrm{~kg}$ foram encontrados valores inferiores na altura do CMJ nas categorias médio/meio-pesado (81$90 \mathrm{~kg} / 90-100 \mathrm{~kg}$ ) quando comparado com as categorias ligeiro/meio-leve $(55-60 \mathrm{~kg} / 60-66 \mathrm{~kg})$ e leve/meio-médio (81-90 kg/90-100 kg), sendo que o percentual de gordura foi significativamente superior nos judocas mais pesados em comparação aos demais. Segundo Tricoli et al. ${ }^{14}$, a gordura corporal representa uma desvantagem no desempenho esportivo, uma vez que náo contribui ativamente para a execução dos movimentos, causando uma sobrecarga negativa para o atleta deslocar-se nas atividades esportivas.

Apesar da importância dos aspectos mencionados, observa-se uma escassez de estudos na literatura analisando os parâmetros de desempenho no salto vertical em judocas de diferentes categorias de peso, considerando a gordura corporal como uma variável interveniente. É extremamente importante que os técnicos e preparadores físicos levem em consideração que os atletas de judô têm características fisiológicas e morfológicas que diferem de acordo com a categoria de peso ${ }^{3}$ e, provavelmente desempenhos diferentes quando realizados exercícios de força e potência.

Com base no exposto, o objetivo deste estudo foi verificar a influência da gordura corporal nos parâmetros cinéticos do salto vertical (altura, potência, força e velocidade) em judocas de diferentes categorias de peso. A hipótese do estudo é de que os atletas de categorias mais pesadas apresentarão menor desempenho nas variáveis de altura e potência quando comparado com judocas de categorias mais leves. No entanto, essa diferença deixará de existir quando retirado o efeito da gordura corporal.

foi de 7,7 \pm 5,3 anos. Com relação à graduação, nove atletas eram faixas amarela, quatro faixas laranja, sete faixas verde, oito faixas roxa, 14 faixas marrom e seis faixas preta. Os atletas do estudo participavam regularmente de competiçóes de nível estadual e alguns de nível nacional. Para evitar distorçóes no perfil antropométrico foi excluída a categoria pesado (acima de 100 $\mathrm{kg}$ ) da amostra. Antecedendo as coletas de dados, os participantes foram esclarecidos sobre os objetivos do estudo e assinaram o termo de consentimento livre e esclarecido. O presente 
estudo foi aprovado pelo Comitê de Ética em Pesquisas com Seres Humanos da Universidade local (parecer 119.014 de 08/10/2012).

\section{Procedimentos}

As coletas foram realizadas na fase de preparação básica de treinamento (início da temporada), sendo que os atletas náo estavam em período de perda rápida de peso. Durante o período das coletas, os atletas treinavam 3-4 vezes na semana com duração de 90 minutos. Os atletas participavam de sessóes de treino tradicionais, incluindo aquecimento (15 min), parte principal composto de treinamento técnico $(35 \mathrm{~min})$ e simulação de luta $(40 \mathrm{~min})^{15}$. Foi recomendado aos atletas se alimentassem com intervalo de pelo menos $1 \mathrm{~h}$ antes das avaliaçóes. As coletas foram realizadas no período vespertino, respeitando a seguinte ordem: 1) Avaliação antropométrica; 2) Avaliaçáo do salto vertical. Todas as avaliaçóes foram realizadas antes das sessóes de treino dos atletas.

\section{Avaliação antropométrica}

As medidas antropométricas de massa corporal e estatura foram realizadas utilizando uma balança digital da marca Toledo ${ }^{\oplus}$ com precisão de $100 \mathrm{~g} \mathrm{e}$ um estadiômetro com precisão de $1 \mathrm{~mm}$, respectivamente. Para as medidas de dobras cutâneas, foi utilizado um adipômetro da marca Cescorf ${ }^{\bullet}$, com precisão de $1 \mathrm{~mm}$. A avaliação foi realizada antes de qualquer esforço físico prévio dos atletas. Todas as medidas foram realizadas por um avaliador experiente (nível 1 da International Society for Advancement in Kinanthropometry - ISAK). Para a medida das dobras cutâneas utilizou-se o procedimento de três medidas sequenciais, sendo considerada para a análise a média. A densidade corporal (DC) foi estimada a partir da equaçáo generalizada proposta por Petroski ${ }^{16}$, a qual considera a medida de quatro dobras cutâneas (tríceps, subescapular, suprailíaca e panturrilha medial) e a idade. A partir da densidade corporal dos sujeitos foi determinado o percentual de gordura $(\% \mathrm{G})$, por meio da equação de SIRI ${ }^{17}$.

\section{Avaliação do salto vertical (CMJ)}

Para avaliar a potência muscular de membros inferiores dos atletas foi utilizado o sal to vertical com contramovimento (CMJ). Para tal, foi utilizada uma plataforma de força (Kistler, Quattro Jump, modelo
9290AD, Winterthur, Switzerland), que consiste em uma plataforma portátil do tipo piezoelétrica, na qual realiza medida apenas da força vertical. As informaçôes adquiridas foram transmitidas via cabo a um computador na frequência de $500 \mathrm{~Hz}$. Para realizar o CMJ, o atleta partiu de uma posiçáo em pé e com as mãos na cintura, disposto sobre a plataforma de força. Posteriormente, executou um contramovimento, que consiste em uma aceleração para baixo do centro de gravidade, flexionando os joelhos até próximo aos $90^{\circ}$. Durante o salto, o tronco manteve-se o mais vertical possível. Os atletas foram familiarizados com os procedimentos do teste realizando aquecimento específico, composto por saltitos e 8-10 saltos submáximos. Após esse procedimento, cada atleta executou três CMJ. A partir da análise da força de reaçáo do solo (FRS), foram analisadas as seguintes variáveis, conforme definido na literatura ${ }^{18-19}$ :

a) Altura do salto: calculada por meio do método da dupla integração da FRS.

b) Força máxima: identificado como o maior valor obtido na fase concêntrica do salto, absoluto e normalizado pela massa corporal.

c) Pico de potência: maior valor da curva obtida a partir da multiplicaçáo da FRS pela velocidade na fase concêntrica do salto, absoluto e normalizado pela massa corporal.

d) Potência média: valor médio da curva obtida a partir da multiplicação da FRS pela velocidade na fase concêntrica do salto, normalizado pela massa corporal.

e) Pico de velocidade: maior valor da velocidade que ocorre imediatamente antes da perda de contato do pé com o solo.

\section{Análise Estatística}

Para a apresentação dos resultados foi utilizada estatística descritiva (média e desvio-padrão) e para testar a normalidade dos dados e dos resíduos foi utilizado o teste de Kolmogorov-Smirnov, os quais foram considerados com distribuição normal para todas as variáveis. Foi utilizada análise de variância (ANOVA one way) com post hoc de Bonferroni para comparar as variáveis do salto vertical (altura, força máxima normalizada e absoluta, pico de potência normalizada e absoluta, potência média e pico de velocidade) entre as categorias de peso. Além disso, foi realizada uma análise de covariância (ANCOVA) com post hoc de Bonferroni para comparar as variáveis do salto vertical entre as categorias de 
peso, tendo o percentual de gordura como covariável. Esta técnica foi utilizada a fim de retirar a influência da gordura corporal na comparação das variáveis analisadas. $\mathrm{O}$ nível de significância adotado foi de $\mathrm{p}<0,05$. As análises foram realizadas no software Statistical Package for Social Sciences (SPSS Inc. v.17.0, Chicago, USA). Além disso, foi calculado o tamanho do efeito (effect size) usando o método do partial eta square $\left(\eta^{2}\right)$ e utilizada a classificação da magnitude dos efeitos proposta por $\operatorname{CoHen}^{20}(<0,2$ pequeno, $0,2-0,8$ moderado e $>$ 0,8 grande). A fim de garantir a representatividade da amostra calculou-se o poder estatístico, sendo que para as variáveis com diferença significativa o poder variou entre $0,73-1,0$, indicando baixo erro tipo II.

\section{Resultados}

No primeiro momento, a fim de controlar uma possível influência do tempo de prática no desempenho do CMJ, foi realizada uma comparação dos valores de tempo de prática entre as três categorias definidas no presente estudo, não sendo encontrada diferença significativa $(F=0,70 ; p=0,50$; pequeno efeito). Dessa forma, foi excluído esse possível fator de interferência nas análises posteriores.

Com relação à análise das variáveis antropométricas, os resultados de acordo as categorias de peso estão apresentados na TABELA 1. Observou-se diferença significativa da massa corporal $(\mathrm{F}=90,23$; $\mathrm{p}<0,05$; grande efeito), estatura $(\mathrm{F}=12,15 ; \mathrm{p}<$ 0,05 ; moderado efeito), soma das dobras ( $\mathrm{F}=34,51$; $\mathrm{p}<0,01$; moderado efeito) e percentual de gordura $(\mathrm{F}=41,75 ; \mathrm{p}<0,05$; moderado efeito $)$ entre as categorias. Analisando os valores de post-hoc, a massa corporal foi superior na categoria $81-90 \mathrm{~kg} / 90-100$ $\mathrm{kg}$, seguida pela $66-73 \mathrm{~kg} / 73-81 \mathrm{~kg}$ e $55-60 \mathrm{~kg} / 60$ $66 \mathrm{~kg}(\mathrm{p}<0,05)$; a estatura foi inferior na categoria $55-60 \mathrm{~kg} / 60-66 \mathrm{~kg}$ quando comparado com as demais $(\mathrm{p}=0,05)$ e; o percentual de gordura foi superior na $81-90 \mathrm{~kg} / 90-100 \mathrm{~kg}$ quando comparado com as demais $(\mathrm{p}<0,05)$.

TABELA 1 - Comparação das variáveis antropométricas entre as categorias de peso.

ANOVA one way com post hoc de Bonferroni $(p<0,05)$; " significativamente diferente das categorias $55-60 \mathrm{~kg} / 60-66$ $\mathrm{kg}$; $\neq$ significativamente diferente das categorias $66-73 \mathrm{~kg} / 73-81 \mathrm{~kg}$; $\S$ significativamente diferente das categorias $66-73 \mathrm{~kg} / 73-81 \mathrm{~kg} \mathrm{e}$ $81-90 \mathrm{~kg} / 90-100 \mathrm{~kg}$

\begin{tabular}{|c|c|c|c|c|}
\hline & $\begin{array}{l}55-60 \mathrm{~kg} / \\
60-66 \mathrm{~kg}\end{array}$ & $\begin{array}{c}66-73 \mathrm{~kg} / \\
73-81 \mathrm{~kg}\end{array}$ & $\begin{array}{l}81-90 \mathrm{~kg} / \\
90-100 \mathrm{~kg}\end{array}$ & $\eta^{2}$ \\
\hline Massa corporal $(\mathrm{kg})$ & $63,19 \pm 5,25 \neq$ & $78,71 \pm 6,63$ & $94,51 \pm 5,55^{*}$ & 0,82 \\
\hline Estatura $(\mathrm{cm})$ & $169,21 \pm 8,21 \S$ & $179,10 \pm 4,58$ & $181,54 \pm 7,80$ & 0,34 \\
\hline Soma das dobras (mm) & $40,05 \pm 11,37$ & $43,48 \pm 9,06$ & $76,46 \pm 13,67^{*}$ & 0,65 \\
\hline Gordura corporal (\%) & $11,87 \pm 2,00$ & $13,09 \pm 2,02$ & $18,73 \pm 1,20 *$ & 0,69 \\
\hline
\end{tabular}

Na TABELA 2 estão apresentados os valores das variáveis do CMJ analisados por categorias de peso. Observou-se diferença significativa nas seguintes variáveis para o grupo $81-90 \mathrm{~kg} / 90-100 \mathrm{~kg}$ - altura $(\mathrm{F}=4,19 ; \mathrm{p}=0,01 ;$ moderado efeito), potência média $(F=4,57 ; p=0,01 ;$ moderado efeito $)$ pico de potência $(\mathrm{F}=3,37 ; \mathrm{p}=0,04$; moderado efeito), pico de velocidade $(\mathrm{F}=4,74 ; \mathrm{p}=0,01$; pequeno efeito), força máxima absoluta $(\mathrm{F}=30,90 ; \mathrm{p}<$ 0,01 ; moderado efeito) e pico de potência absoluta $(\mathrm{F}=35,43 ; \mathrm{p}<0,01 ;$ moderado efeito $)$. Não foi encontrada diferença na força máxima normalizada ( $\mathrm{F}=2,95 ; \mathrm{p}=0,06$; pequeno efeito). Com relação à análise do post hoc, todas as variáveis foram inferiores no grupo $81-90 \mathrm{~kg} / 90-100 \mathrm{~kg}$ em comparação aos demais grupos, com exceção da força máxima e pico de potência absolutos, os quais foram superiores no grupo $81-90 \mathrm{~kg} / 90-100$ $\mathrm{kg}$ e $66-73 \mathrm{~kg} / 73-81 \mathrm{~kg}$ quando comparado com $55-60 \mathrm{~kg} / 60-66 \mathrm{~kg}$.

A TABELA 3 mostra a comparação das variáveis do $\mathrm{CMJ}$ entre as categorias de peso com o percentual de gordura como covariável. Não foram verificadas diferenças significativas nas variáveis - altura $(\mathrm{F}=$ 0,$21 ; \mathrm{p}=0,81$; pequeno efeito), força máxima relativa $(\mathrm{F}=0,22 ; \mathrm{p}=0,80$; pequeno efeito), potência média ( $\mathrm{F}=0,09 ; \mathrm{p}=0,91$; pequeno efeito), pico de potência relativo $(F=0,36 ; p=0,70$; pequeno 
efeito) e pico de velocidade ( $\mathrm{F}=0,25 ; \mathrm{p}=0,78$; pequeno efeito). Nas variáveis força máxima absoluta ( $\mathrm{F}=11,83 ; \mathrm{p}<0,01 ;$ moderado efeito) e pico de potência absoluta $(\mathrm{F}=13,54 ; \mathrm{p}<0,01$; moderado efeito) foi observado que a categoria $55-60 \mathrm{~kg} / 60$ $66 \mathrm{~kg}$ foi significativamente diferente das demais.

TABELA 2 - Comparação das variáveis do CMJ entre as categorias de peso.

\begin{tabular}{|c|c|c|c|c|}
\hline & $\begin{array}{l}55-60 \mathrm{~kg} / \\
60-66 \mathrm{~kg}\end{array}$ & $\begin{array}{l}66-73 \mathrm{~kg} / \\
73-81 \mathrm{~kg}\end{array}$ & $\begin{array}{l}81-90 \mathrm{~kg} / \\
90-100 \mathrm{~kg}\end{array}$ & $\eta^{2}$ \\
\hline Altura $(\mathrm{cm})$ & $46,78 \pm 6,05$ & $47,42 \pm 4,39$ & $42,11 \pm 4,11 *$ & 0,26 \\
\hline Força máxima (N.kg-1) & $24,14 \pm 2,99$ & $24,27 \pm 2,40$ & $22,21 \pm 1,30$ & 0,12 \\
\hline Força máxima $(\mathrm{N})$ & $1536,57 \pm 177,29 \$$ & $1932,02 \pm 255,00$ & $2121,35 \pm 202,64 \neq$ & 0,58 \\
\hline Potência Média (W.kg-1) & $28,52 \pm 4,16$ & $29,02 \pm 3,28$ & $25,34 \pm 1,98^{*}$ & 0,27 \\
\hline Pico de Potência (W.kg-1) & $49,81 \pm 5,56$ & $50,38 \pm 3,91$ & $45,96 \pm 4,63 *$ & 0,23 \\
\hline Pico de Potência (W) & $3080,08 \pm 423,78 \rrbracket$ & $4002,69 \pm 400,17$ & $4372,39 \pm 399,30 \neq$ & 0,61 \\
\hline Pico de Velocidade (m.s-1) & $2,77 \pm 0,18$ & $2,79 \pm 0,150$ & $2,61 \pm 0,14 *$ & 0,17 \\
\hline
\end{tabular}

ANOVA one way com post hoc de Bonferroni $(p<0,05)$; * significativamente diferente das categorias $55-60 \mathrm{~kg} / 60$ $66 \mathrm{~kg}$ e $66-73 \mathrm{~kg} / 73-81$ $\mathrm{kg}$; ${ }^{*}$ significativamente diferente das categorias $55-60 \mathrm{~kg} / 60-66 \mathrm{~kg}$; ssignificativamente diferente das categorias $66-73 \mathrm{~kg} / 73-81 \mathrm{~kg} \mathrm{e}$ $81-90 \mathrm{~kg} / 90-100 \mathrm{~kg}$.

TABELA 3 - Comparação das variáveis do CMJ entre as categorias de peso com o percentual de gordura como covariável.

\begin{tabular}{|c|c|c|c|c|}
\hline & $\begin{array}{l}55-60 \mathrm{~kg} / \\
60-66 \mathrm{~kg}\end{array}$ & $\begin{array}{l}66-73 \mathrm{~kg} / \\
73-81 \mathrm{~kg}\end{array}$ & $\begin{array}{l}81-90 \mathrm{~kg} / \\
90-100 \mathrm{~kg}\end{array}$ & $\eta^{2}$ \\
\hline Altura $(\mathrm{cm})$ & $46,83 \pm 6,22$ & $46,92 \pm 4,43$ & $41,40 \pm 3,60$ & 0,11 \\
\hline Força máxima (N.kg-1) & $24,29 \pm 3,01$ & $24,34 \pm 2,39$ & $22,16 \pm 1,33$ & 0,12 \\
\hline Força máxima $(\mathrm{N})$ & $1529,24 \pm 179,27 *$ & $1917,10 \pm 254,35$ & $2097,13 \pm 201,83$ & 0,39 \\
\hline Potência Média (W.kg-1) & $28,63 \pm 4,25$ & $28,50 \pm 3,04$ & $25,32 \pm 2,08$ & 0,05 \\
\hline Pico de Potência (W.kg-1) & $49,87 \pm 5,71$ & $50,21 \pm 3,91$ & $45,52 \pm 4,97$ & 0,02 \\
\hline Pico de Potência (W) & $3146,06 \pm 408,50 *$ & $3945,07 \pm 369,00$ & $3669,68 \pm 618,00$ & 0,42 \\
\hline Pico de Velocidade (m.s-1) & $2,77 \pm 0,19$ & $2,7814 \pm 0,150$ & $2,60 \pm 0,14$ & 0,01 \\
\hline
\end{tabular}

ANCOVA com post hoc de Bonferroni $(p<0,05)$; * significativamente diferente das categorias $66-73 \mathrm{~kg} / 73-81 \mathrm{~kg} \mathrm{e}$ $81-90 \mathrm{~kg} / 90-100 \mathrm{~kg}$.

\section{Discussão}

Este estudo investigou a influência da gordura corporal no desempenho do salto vertical em judocas de diferentes categorias de peso. A hipótese do estudo foi aceita, uma vez que os atletas de categorias mais pesadas apresentaram menor desempenho em quase todas as variáveis do CMJ (com exceção da força máxima), quando comparado com judocas de categorias mais leves. Porém, essas diferenças deixaram de existir quando usado o percentual de gordura como covariável, ou seja, retirando seu efeito.

O salto vertical é conhecido por ser um importante método no diagnóstico do desempenho da potência muscular em diversos esportes que envolvem açóes explosivas, sendo inferido prin- cipalmente pela altura do salto e potência ${ }^{9}$. Em esportes de combate, diversos estudos têm utilizado o desempenho no salto vertical com o intuito de verificar efeito de treinamento ${ }^{21}$, discriminar o nível competitivo ${ }^{22-23}$, sexo e categoria de peso ${ }^{11,24}$ e como marcador de fadiga antes e após sequência de lutas ${ }^{25-28}$, como sintetizado na TABELA 4. Os valores de altura do salto obtido na presente investigação, considerando a média das categorias $(45,4$ $\pm 2,4 \mathrm{~cm}$ ), é similar a alguns estudos ${ }^{11,27}$ e superior aos demais ${ }^{21-22,28}$ que analisaram esta variável para a mesma faixa etária e nível competitivo. Nenhum dos estudos analisados comparou o salto vertical entre categorias, considerando as variáveis antropométricas dos atletas. 
TABELA 4 - Síntese de alguns estudos que avaliaram o salto vertical em esportes de combate para atletas do sexo masculino.

$\mathrm{CMJ}$ : countermovement jump; SJ: squat jump; mas: masculino; fem: feminino; N: não-elite; $E$ : elite; S: sênior, J: júnior.

\begin{tabular}{|c|c|c|c|c|c|}
\hline Autores & Sujeitos & $\begin{array}{l}\text { Idade } \\
\text { (anos) }\end{array}$ & Salto & Modalidade & Resultados \\
\hline Detanico et al. ${ }^{11}$ & $\begin{array}{c}18 \text { atletas } \\
\text { brasileiros - } \\
\text { nível estadual }\end{array}$ & $20,6 \pm 1,8$ & $\mathrm{CMJ}$ & Judô & $44,8 \pm 4,4 \mathrm{~cm}$ \\
\hline Detanico et al. ${ }^{27}$ & $\begin{array}{l}20 \text { atletas } \\
\text { brasileiros - } \\
\text { nível estadual/ } \\
\text { nacional }\end{array}$ & $20,7 \pm 4,6$ & $\mathrm{CMJ}$ & Judô & $\begin{array}{l}\text { Pré-luta: } 45,4 \pm 5,2 \mathrm{~cm} \\
\text { Pós-luta } 1: 44,9 \pm 5,6 \mathrm{~cm} \\
\text { Pós-luta 2: } 43,7 \pm 5,8 \mathrm{~cm} \\
\text { Pós-luta } 3: 43,9 \pm 5,1 \mathrm{~cm}\end{array}$ \\
\hline $\begin{array}{l}\text { Torres-LUQUe } \\
\text { et al. }{ }^{24}\end{array}$ & $\begin{array}{l}146 \text { atletas } \\
\text { espanhóis } \\
\text { não-elite }\end{array}$ & $14,7 \pm 1,1$ & $\mathrm{CMJ}$ & Judô & $30,8 \pm 10,5 \mathrm{~cm}$ \\
\hline $\begin{array}{l}\text { ZAGGELIDIS e } \\
\text { LAZARIDIS }^{23}\end{array}$ & $\begin{array}{l}20 \text { atletas } \\
\text { Grécia elite/ } \\
\text { náo-elite }\end{array}$ & $21,5 \pm 4,6$ & $\begin{array}{l}\text { CMJ } \\
\text { e SJ }\end{array}$ & Judô & $\begin{array}{c}\text { CMJ N: } 24,8 \pm 3,8 \mathrm{~W} / \mathrm{kg} \\
\text { CMJ E: } 26,6 \pm 4,3 \mathrm{~W} / \mathrm{kg} \\
\text { SJ E: } 26,6 \pm 4,3 \mathrm{~W} / \mathrm{kg} \\
\text { SJ N: } 22,2 \pm 4,1 \mathrm{~W} / \mathrm{kg}\end{array}$ \\
\hline Monteiro et al. ${ }^{22}$ & $\begin{array}{l}76 \text { atletas elite } \\
\text { sênior/júnior } \\
\text { Portugal, Tuni- } \\
\text { sa, Brasil, Fran- } \\
\text { ça e Espanha. }\end{array}$ & $21,9 \pm 3,4$ & $\begin{array}{l}\text { CMJ } \\
\text { e SJ }\end{array}$ & Judô & $\begin{array}{l}\text { CMJ S: } 41,2 \pm 6,7 \mathrm{~cm} \\
\text { CMJ J: } 42,7 \pm 7,2 \mathrm{~cm} \\
\text { SJ S: } 41,2 \pm 6,7 \mathrm{~cm} \\
\text { SJ J: } 44,5 \pm 5,8 \mathrm{~cm}\end{array}$ \\
\hline FrANCHINI et al. ${ }^{21}$ & $\begin{array}{l}10 \text { atletas bra- } \\
\text { sileiros sênior - } \\
\text { nível estadual }\end{array}$ & $23 \pm 2$ & $\mathrm{CMJ}$ & Judô & $\begin{array}{l}\text { Pré: } 35,4 \pm 4,2 \mathrm{~cm} \\
\text { Pós: } 34,8 \pm 4,1 \mathrm{~cm}\end{array}$ \\
\hline KraEmer et al. ${ }^{26}$ & $\begin{array}{l}12 \text { atletas } \\
\text { americanos } \\
\text { profissionais }\end{array}$ & $19,3 \pm 1,2$ & $\mathrm{CMJ}$ & $\begin{array}{l}\text { Luta olímpica } \\
\text { estilo livre }\end{array}$ & $4758,5 \pm 361,5 \mathrm{~W}$ \\
\hline BARBAS et al. ${ }^{25}$ & $\begin{array}{l}12 \text { atletas } \\
\text { Grécia - nível } \\
\text { nacional/ } \\
\text { internacional }\end{array}$ & $22,1 \pm 1,3$ & $\mathrm{CMJ}$ & $\begin{array}{l}\text { Luta greco- } \\
\text {-romana }\end{array}$ & $41,7 \pm 1,9 \mathrm{~cm}$ \\
\hline ANDREATO et al. ${ }^{28}$ & $\begin{array}{l}10 \text { atletas } \\
11 \pm 4 \text { anos de } \\
\text { prática regular }\end{array}$ & $28 \pm 4$ & $\mathrm{CMJ}$ & Jiu-jitsu & $41 \pm 6 \mathrm{~cm}$ \\
\hline
\end{tabular}

No presente estudo, observou-se que os judocas de categorias mais leves apresentam maiores valores na altura do salto, potência média e pico de potência quando comparado às demais categorias (TABELA 2). Detanico et al. ${ }^{11}$ encontraram resultados semelhantes, mostrando que judocas de categorias mais leves (menor massa corporal) apresentaram maiores valores de altura do salto e potência quando comparado aos judocas mais pesados. Um fator que pode explicar essa diferença entre categorias de peso é o maior pico de velocidade atingido na fase concêntrica do salto pelos atletas mais leves, visto que o mesmo é considerado o principal determinante da potência e da altura do salto ${ }^{18,29}$. Assim, os atletas mais leves conseguem aplicar maiores magnitudes de velocidade na fase concêntrica do salto do que os atletas mais pesados, resultando em maior potência e altura do salto.

Outro fator importante que pode justificar os achados encontrados é a eficiência de alguns mecanismos musculoelásticos presentes na ação muscular durante o salto, tal como a rapidez na transição entre as fases excêntrica e concêntrica. De acordo com Komi e Gollhofer ${ }^{30}$, essa transição deve ser realizada em um breve espaço de tempo, a fim de evitar a dissipação da energia elástica acumulada nas estruturas musculotendíneas. Esse mecanismo pode não ser táo eficiente nos atletas mais pesados, em função da maior sobrecarga (massa corporal) durante a fase excêntrica em comparação a atletas 
mais leves, dificultando o processo de transição entre as fases do movimento e a consequente utilização de energia elástica para a produção de potência.

Com relação à força e potência absolutas, foram reportados valores superiores nos judocas mais pesados comparados aos leves. Por possuírem maior massa corporal, os atletas conseguiram gerar maiores níveis de força e potência absoluta durante o salto. Estudo prévio já havia verificado relação inversa entre força máxima absoluta obtida no CMJ e massa corporal (indicador de categorias de peso) ${ }^{11}$. Por outro lado, quando comparada a força máxima normalizada náo foi encontrada diferença entre as categorias, indicando que atletas leves e pesados não diferem na capacidade de gerar força por quilo de massa. Acredita-se que o fato dos judocas de categorias mais pesadas terem maior gordura corporal (TABELA 1) possa explicar esses resultados.

Um achado inédito deste estudo foi que quando realizada a comparaçáo das variáveis do CMJ entre as categorias de peso usando o percentual de gordura como covariável, ou seja, retirando seu efeito, todas as diferenças desapareceram, com exceção da força e potência absolutas (TABELA 3). Esses resultados sugerem que a gordura corporal em nível elevado pode ser considerada umas das variáveis que influenciaram negativamente o desempenho no salto vertical nos atletas deste estudo. $\mathrm{O}$ excesso de gordura corporal também tem sido associado com a diminuição da performance em teste anaeróbio (Wingate) em judocas de elite ${ }^{31}$. Ainda, Katralli e GoUdAR ${ }^{32}$ encontraram correlação negativa entre percentual de gordura e o número de arremessos no SJFT, indicando que o excesso de gordura corporal prejudica esforços neuromusculares exigidos em ações específicas do judô.

Analisando força e potência absolutas, observouse que os atletas das categorias mais leves (55-60 $\mathrm{kg} / 60-66 \mathrm{~kg}$ ) apresentaram valores inferiores quando comparado aos atletas mais pesados. Mesmo com percentual de gordura menor, os judocas mais leves possuem também menor massa corporal quando comparado aos atletas das categorias mais pesadas. Tendo em vista que força depende da massa e da aceleração $(\mathrm{F}=\mathrm{m}$.a) e a potência da força e da velocidade $(\mathrm{P}=\mathrm{F} . \mathrm{V})$, os judocas mais leves conseguem aplicar menores magnitudes de força e potência absolutas na execução do salto.

É importante destacar algumas limitaçóes do presente estudo, tais como o agrupamento em três categorias de peso, ao invés de utilizar as sete categorias do judô. Esse procedimento foi realizado a fim de garantir uma amostra adequada em cada categoria, diminuindo assim os erros típicos.

Em síntese, pode-se concluir que judocas de categorias mais leves apresentaram valores superiores na maioria das variáveis do CMJ (altura do salto, potência e velocidade) quando comparados com atletas de categorias mais pesadas. Esses resultados parecem ser influenciados pelo menor percentual de gordura dos judocas de categorias mais leves.

\section{Aplicações práticas}

Recomenda-se que os técnicos e preparadores físicos levem em consideração as características antropométricas, em especial o percentual de gordura, no momento de planejar as cargas de treino, a fim de minimizar a influência desta variável em açóes que envolvam movimentos explosivos nos membros inferiores, como é o caso de diversas técnicas de projeção no judô. Sugere-se também adicionar sessões de treino que tenham como foco a diminuição da gordura corporal e aumento da massa livre de gordura em judocas de categorias pesadas. Nesse sentido, programas de treinamento intervalado de alta intensidade surgem como uma opção interessante, uma vez que têm sido relacionados com a diminuição da gordura visceral e abdominal e aumento da massa livre de gordura em jovens com sobrepeso ${ }^{33-34}$. Treinamentos contínuos também podem ser incluídos, principalmente na fase de preparação básica dos atletas.

Em outra perspectiva e sabendo da importância da avaliação do salto vertical para a inferência da potência muscular em atletas de judô, ressalta-se que instrumentos mais simples e com custos mais baixos em relação à plataforma força podem ser utilizados, como por exemplo, tapetes de contato ${ }^{21,24-25,28} \mathrm{e}$ fitas métricas ${ }^{35}$. Além do salto vertical, o teste de salto horizontal também pode ser utilizado a fim de identificar efeitos de treinamento de potência ao longo da periodizaçãa ${ }^{34}$. 


\begin{abstract}
Influence of body fat on countermovement jump performance in judo athletes from different weight categories
\end{abstract}

This study aimed to verify the influence of body fat on kinetic parameters of vertical jump in judo athletes from different weight categories. The study included 48 male judokas grouped by categories: $55-60 \mathrm{~kg} / 60-$ $66 \mathrm{~kg}$ (light/half-light), 66-73 kg/73-81 kg (light/half-middle) and 81-90 kg/90-100 kg (middle/half-heavy weight). Judokas performed vertical jumps such as the countermovement jump (CMJ) on a force platform, and the following performance variables were analyzed: jump height and power, maximum power and peak velocity. Analysis of variance (ANOVA) and analysis of covariance (ANCOVA) - body fat as covariate - were used with the level of significance set at $p<0.05$. The main results showed that athletes in heavier categories (81-90 kg/90-100 kg) showed higher body fat $(p<0.05)$ and sum of skinfold thickness $(p<0.05)$ when compared to lighter categories $(55-60 \mathrm{~kg} / 60-66 \mathrm{~kg}$ and $66-73 \mathrm{~kg} / 73-81 \mathrm{~kg})$. Lower performance was found in almost all CMJ variables when compared to judokas from lighter categories, with highlight for jump height $(55-60 \mathrm{~kg} / 60-66 \mathrm{~kg}=46.78 \pm 6.05 \mathrm{~cm} ; 66-73 \mathrm{~kg} / 73-81 \mathrm{~kg}=47.42 \pm 4.39 \mathrm{~cm} ; 81-90$ $\mathrm{kg} / 90-100 \mathrm{~kg}=42.11 \pm 4.11 ; \mathrm{p}<0.05)$. However, when the effect of body fat was removed, the differences disappeared $(p>0.05)$. We concluded that the judokas from lighter categories showed higher values in the most of CMJ variables (jump height, power and velocity) when compared with judokas from heavier categories. This seems to be influenced by lower body fat in lighter judo athletes.

KEYwORDS: Combat sports; Stretch-shortening cycle; Countermovement jump; Muscle power; Body composition.

\title{
Referências
}

1. Miarka B, Del Vecchio FB, Franchini E. Acute effects and postactivation potentiation in the Special Judo Fitness Test. J Strength Cond Res 2011;25(2):427-431.

2. Franchini E, Sterkowicz-Przybycien K, Takito MY. Anthropometrical profile of judo athletes: comparative analysis between weight categories. Int J Morphol 2014;32(1):36-42.

3. Franchini E, Matsushigue KA, Vecchio FB, Artioli GG. Physiological profiles of elite judo athletes. Sports Med 2011;41(2):147166.

4. Filaire E, Sagnol M, Ferrand C, Maso F, Lac G. Psychophysiological stress in judo athletes during competitions. J Sports Med Phys Fitness 2001;41(2):263-268.

5. Franchini E, Artioli GG, Brito CJ. Judo combat: time-motion analysis and physiology. Int J Perform Analysis Sport 2013;13:624-641.

6. International Judo Federation (IJF). IJF Referee Rules. [acesso em 11 nov. 2017]. Disponível em: http://www.intjudo. eu/Rules/IJF_Referee_Rules.

7. Marcon G, Franchini E, Jardim JR, Barros Neto TL. Structural analysis of action and time in sports: Judo. J Quant Analysis Sport 2010;6(4):1-13.

8. Detanico D, Dal Pupo J, Franchini E, Santos S. Relationship of aerobic and neuromuscular indexes with specific actions in judo. Sci Sport 2012;1(27):16-22.

9. Markovic G, Jaric S. Is vertical jump height a body size independent measure of muscle power? J Sports Sci 2007;25(12):1355-1363.

10. Duncan MJ, Hankey J, Lyons M, James RS, Nevill AM. Peak power prediction in junior basketballers: comparing linear and allometric models. J Strength Cond Res 2013;27(3):597-603.

11. Detanico D, Dal Pupo J, Santos SG, Arins FB. Strength parameters in judo athletes: an approach using hand dominance and weight categories. Hum Movem 2012;13(4):330-336.

12. Franchini E, Nunes AV, Moraes JM, Del Vecchio FB. Physical fitness and anthropometrical profile of the Brazilian male judo team. J Physiol Anthropol 2007;26:59-67.

13. Detanico D, Dal Pupo J, Santos SG. Comparação de índices neuromusculares e fisiológicas de judocas em diferentes categorias de peso. Rev Educ Fís/UEM 2011;22(3):433-440. 
14. Tricoli VA, Barbanti VJ, Shinzato GT. Potência muscular em jogadores de basquetebol e voleibol. Rev Paul Educ Fís 1994;8(2):14-25.

15. Brito CJ, Gatti K, Mende, EL, Nobrega OT, Marins JCB, Franchni E. Carbohydrate intake and immunosuppression during judo training. Med Sport 2011;64(4):393-408.

16. Petroski EL, Pires-Neto CS. Validação de equações antropométricas para estimativa da densidade corporal em homens. Rev Bras Atividade Fis Saúde 1996;1(3)5-14.

17. Siri WE. Body composition from fluids spaces and density: analyses of methods. In: Techniques for measuring body composition. Washington, DC: National Academy of Science and Natural Resource Council; 1961.

18. Dal Pupo J, Detanico D, Santos SG. Kinetic parameters as determinants of vertical jump performance. Braz J Kinanthropom Hum Performance 2012;14(1):41-51.

19. Linthorne NP. Analysis of standing vertical jumps using a force platform. Am J Phys 2001;69(11):1198-1204.

20. Cohen J. Statistical Power Analysis for the Behavioral Sciences. Hillsdale: Lawrence Earlbaum Associates; 1988.

21. Franchini E, Del Vecchio FB, Ferreira Julio U, Matheus L, Candau R. Specificity of performance adaptations to a periodized judo training program. Rev Andal Med Deporte 2015;8(2):67-72.

22. Monteiro LF, Massuça LM, García JG, Carratala V, Proença J. Plyometric muscular action tests in judo- and non-judo athletes. Isok Exer Scie 2011;19(4):287-293.

23. Zaggelidis G, Lazaridis S. Muscle activation profiles of lower extremities in different throwing techniques and in jumping performance in elite and novice Greek judo athletes. J Human Kinet 2013;37(5):63-70.

24. Torres-Luque G, Hernandez-Garcia R, Garatachea N, Nikolaidis PT. Anthropometric characteristics and neuromuscular function in young judo athletes by sex, age and weight category. Sport Sci Health 2015;11(1):117-124.

25. Barbas I, Fatouros IG, Douroudos II, et al. Physiological and performance adaptations of elite Greco-Roman wrestlers during a one-day tournament. Eur J Appl Phys 2011;111(7):1421-1136.

26. Kraemer WJ, Fry AC, Rubin MR, et al. Physiological and performance responses to tournament wrestling. Med Sci Sports Exerc 2001;33(8):1367-1378.

27. Detanico D, Dal Pupo J, Franchini E, dos Santos SG. Effects of successive judo matches on fatigue and muscle damage markers. J Strength Cond Res 2015;29(4):1010-1016.

28. Andreato LV, Julio UF, Panissa VL, et al. Brazilian jiu-jitsu simulated competition part II: Physical performance, time-motion, technical-tactical analyses, and perceptual responses. J Strength Cond Res 2015;29(7):2015-2025.

29. Yamauchi J, Ishii N. Relations between force-velocity characteristics of the knee-hip extension movement and vertical jump performance. J Strength Cond Res 2007;21(3):703-709.

30. Komi PV, Gollhofer A. Stretch reflex can have an important role in force enhancement during SSC-exercise. J Appl Biomech 1997;13(4):451-460.

31. Kim J, Cho HC, Jung HS, Yoon JD. Influence of performance level on anaerobic power and body composition in elite male judoists. J Strength Cond Res 2011;25(5):1346-1354.

32. Katralli J, Goudar SS. Anthropometric profile and Special Judo Fitness levels of Indian Judo Players. Asian J Sports Med 2012;3(2):113-118.

33. Boutcher SH. High-intensity intermittent exercise and fat loss. J Obes 2011;2011:868305.

34. Stiegle P, Cunliffe A. The role of diet and exercise for the maintenance of fat-free mass and resting metabolic rate during weight loss. Sports Med 2006;36(3):239-262.

35. Franchini E, Branco BM, Agostinho MF, Calmet M, Candau R. Influence of linear and undulating strength periodization on physical fitness, physiological, and performance responses to simulated judo matches. J Strength Cond Res 2015;29(2):358-367.

ENDEREÇO DE CORRESPONDÊNCIA Marina Saldanha da Silva Athayde Departamento de Educação Física - Campus Trindade Universidade Federal de Santa Catarina CEP 88040-900

Florianópolis - Santa Catarina - Brasil E-mail: marinasaldanha.sa@hotmail.com
Submetido: 24/07/2015

$1^{\mathrm{a}}$. Revisão: 08/09/2015

$2^{\text {a }}$. Revisão: 09/10/2015

$3^{\text {a }}$. Revisão: 12/11/2015

$4^{\mathrm{a}}$. Revisão: $23 / 11 / 2015$

Aceito: $29 / 12 / 2015$ 
